\title{
Antiretroviral therapy programme outcomes in Tshwane district, South Africa: A 5-year retrospective study
}

\author{
N Mlangeni, MPH, BCur; F Senkubuge, MB ChB, MMed (Public Health), FCPHM (SA) \\ School of Health Systems and Public Health, Faculty of Health Sciences, University of Pretoria, South Africa
}

Corresponding author: N Mlangeni (milo.mlangeni@yahoo.com)

\begin{abstract}
Background. Scaling up of antiretroviral therapy (ART) in South Africa (SA) has resulted in an increase in the number of patients on the national ART programme and an increased workload for ART service providers nationwide.

Objectives. To ascertain patient retention on ART after 5 years on treatment in one district of Gauteng Province, SA, establish the number of patients who remained alive on ART after 5 years of treatment, and identify patient-related factors that contributed towards the outcome of each indicator. Methods. A retrospective cohort study of patients initiated on highly active antiretroviral therapy (HAART) between January and March 2007 was carried out. A sample of 381 patients was randomly selected from 1004 records, and their records were reviewed for visits over the previous 60 months. Summary statistics, Pearson's $\chi^{2}$ test and linear regression tests were performed.

Results. Of 381 patients, 156 (40.9\%) remained alive and active on HAART at their initial sites. The overall mortality rate was $5.0 \%$ and the rate of long-term retention in care was $57.4 \%$, excluding those transferred to another site. After 6 months on HAART the mean rise in CD4 count was 113 cells $/ \mu \mathrm{L}$, and after 60 months it was 288 cells $/ \mu \mathrm{L}$. Viral load suppression to $<400 \mathrm{copies} / \mathrm{mL}$ was achieved in $74.0 \%$ of patients at 6 months and $91.0 \%$ at 60 months.

Conclusions. Immunological and virological outcomes after 5 years on treatment were good. Both these positive outcomes showed that the ART programme was a success. Improved data quality and patient follow-up will further strengthen programme outcomes.

S Afr Med J 2016;106(4):365-368. DOI:10.7196/SAMJ.2016.v106i4.9375
\end{abstract}

South Africa (SA) has the largest national antiretroviral therapy (ART) programme in the world and is one of the countries with a high HIV prevalence. ${ }^{[1]}$ Response to ART depends on various factors, such as gender, age, psychosocial support and disease stage on initiation. ${ }^{[2,3]}$ The main outcomes of the ART programme are improved quality of life and reduced mortality. ${ }^{[4,5]}$

As a result of positive outcomes of ART programmes globally there have been several initiatives for rapid scale-up of ART in resourcelimited countries, including SA. Scaling up of ART in SA has meant an increase in the number of patients on ART and an increased workload for ART service providers. The number of patients who need ART will continue to increase, as the eligibility criteria for ART are continually reviewed to be in line with World Health Organization (WHO) guidelines and up-to-date research findings. ${ }^{[6]}$

The increased workload on health providers resulting from programme scale-up may result in poor programme outcomes. ${ }^{[1,7]}$ While the ART programme is expanding in SA, it is therefore important to monitor the programme on a long-term basis by following cohort outcomes.

\section{Objectives}

To establish the number of patients who remained alive on ART after 5 years of treatment and identify patient-related factors that contributed to the outcome of each indicator. The outcomes measured were retention in care, immunity gain and viral suppression. Evaluation of the outcome measures provides an important means of assessing ART programmes and facilitates development of optimal models of care in resource-limited settings. ${ }^{[8]}$

\section{Methods}

Study setting and inclusion criteria

The study took place in Tshwane district, Gauteng Province, SA. Five facilities participated, and the basis of selection was that they were initiating patients on ART in January 2007 and already had an electronic database. A SOZO database, which has records of all patients on ART in the participating facilities, was used as a data source.

\section{Routine HIV care and clinic visits}

Patients are referred to the ART programme when they are eligible for highly active antiretroviral therapy (HAART), which at the time of the study was when they have a CD4 count $<200$ cells $/ \mu \mathrm{L}$ or met the other criteria for ART initiation. ${ }^{[9]}$ (The SA ART initiation eligibility criteria recently changed to a CD4 count of 350 instead of 200 cells $/ \mu \mathrm{L}$.) Once patients are initiated on HAART they visit the clinic every month to collect medication. A CD4 count is one of the tests done on baseline, and then routine blood tests, including CD4 count and viral load (VL), are done after 6 months. Thereafter these are repeated annually from the date of initiation.

\section{Outcome definitions}

Adverse outcomes combined death, loss to follow-up, default and stopping treatment. Loss to follow-up was defined as patients who had not collected their medication for $>90$ days. Baseline blood tests (CD4 count) were those done before the patient started ART. The VL was considered to be suppressed if it was $<400$ copies $/ \mathrm{mL}$ at 6 months and at the end of 60 months of treatment. Immunological response was measured as the $\mathrm{CD} 4$ count change from baseline. ${ }^{[9]}$

\section{Design}

A retrospective analytical cohort study design was used. The cohort consisted of all patients who had started ART between January and March 2007. These patients had completed 60 months (5 years) on treatment at the end of March 2012.

\section{Sampling}

It has been reported that non-retention of patients on ART in the clinics of SA and the sub-Saharan region ranges from $30 \%$ to $40 \%{ }^{[1,4,1,0]}$ Retention 
rates are usually reported over periods of 2 3 years, and rates would be expected to be higher over the longer term. Assuming a rate of $40-50 \%$ for the purposes of sample size calculations, $45 \%$ was used as a non-retention rate. A margin of error of $5 \%$ was used at a 95\% compliance level, which led to the sample size of 381 records. Simple random sampling was done using Excel commands, where all 1044 patient records from five facilities were listed on one Excel spreadsheet and random selection of 381 records was made.

\section{Statistical analysis}

Summary statistics were used for gender and age at initiation, and medians to describe CD4 counts at baseline and at follow-up. Patient ages were categorised into four groups (Table 1), and a $\chi^{2}$ test was generated to analyse CD4 count in relation to age. To examine the impact of gender on CD4 count change, a simple linear regression test was performed.

Adverse outcomes, i.e. death, default, loss to follow-up or stopping ART, were combined because of the small numbers of each outcome. Multivariate logistic regression was performed to determine the effect of gender on adverse outcomes, and Pearson's $\chi^{2}$ test to examine the effect of age and baseline CD4 count on adverse outcomes. Logistic regression analysis was performed to determine the effect of gender, age and $\mathrm{CD} 4$ count on the adverse outcomes.

CD4 counts at baseline and 6 months were compared using a paired $t$-test. Linear regression analysis was used to examine the effect of gender, age and baseline CD4 count on changes in CD4 counts at 6 months and 60 months, and logistic regression analysis to determine effects of gender and age on VL at 6 months and 60 months.

\section{Results}

\section{Baseline characteristics}

A total of 381 patients were randomised from 1044 patients initiated on ART between January and March 2007 at the five facilities in Tshwane district that participated in the study. Of the 381 patients, 251 (65.9\%) were females and 130 (34.1\%) males. The mean age at which ART was started was 35 years, and the mean baseline CD4 count was 126 cells $/ \mu \mathrm{L}$. Only 60 records had baseline and follow-up CD4 counts, and those were the ones measured and compared. Age group (Table 1) was found to have no effect on baseline CD4 count. Males were found to have started ART at a mean of 3.4 years older than females, which is a significant difference $(p<0.005)$. They also came to the clinics with more advanced disease than females, as indicated by CD4 those of females, also a significant difference $(p<0.004)$.

\section{Adverse outcomes: death, loss to follow-up, default and stopping treatment}

A $\chi^{2}$ test showed that $28.6 \%$ of females and $33.8 \%$ of males had adverse outcomes. This difference does not have statistical significance $(p>0.05)$. The findings were similar when multivariate logistic regression analysis was used, males being 1.3 times more likely to have an adverse outcome; again this was not significant $(p=0.3$ )

While age was not found to affect the rate of adverse outcomes $(p=0.973)$, a low CD4 count was found to have a significant impact ( $p=0.025$ ). However, when examining loss to follow-up as an adverse event on its own, the effects of CD4 count $(p=0.609)$, age $(p=0.722)$ and gender $(p=0.985)$ were not found to be significant.

\section{Treatment outcomes: retention in care, $\mathrm{CD} 4$ change and viral suppression}

Of the 381 patients who started treatment in January - March 2007, 156 (40.9\%) remained in care (Table 2). However, 109 (28.6\%) of the 381 patients had been transferred out, so if these patients are considered as still in care, $69.5 \%$ remained in care. Removing transferred-out patients from the total of 381 leaves 272 patients, giving a retention rate of $57.4 \%$. Since we do not know how many transferredout patients were still on treatment, the latter figure is the most appropriate.

Looking only at the CD4 counts of the 60 patients who had both baseline and 6-month results, there was a significant change between the baseline and 6-month counts (Figs 1 and 2). The mean CD4 count at 6 months was 238 cells/ $\mu \mathrm{L}$, a significant rise of 112 cells $/ \mu \mathrm{L}$ compared with baseline $(p=0.000)$. There were gender differences in CD4 count, the rise for males being counts on average 59 cells $/ \mu \mathrm{L}$ lower than

54 cells/ $\mu \mathrm{L}$ less than that for females; however, this difference was not statistically significant $(p>0.005)$.

Patient age groups had varying influences on the CD4 count change from baseline to 6 months. The 29 - 35-year age group had a

Table 1. Baseline characteristics of the study subjects ${ }^{\star}$

Characteristic

Gender, $n / N(\%)$

Females

251/381 (65.8)

Males

130/381 (34.1)

Age (years), median

$35(50)$

(IQR)

Age categories (years),

$n / N(\%)$

$0-28$

90/368 (23.8)

$29-35$

96/368 (25.4)

$36-42$

95/368 (25.1)

$>42$ $87 / 368(22.8)$

CD4 count (cells $/ \mu \mathrm{L})$ 159

mean

$\mathrm{CD} 4$ category (cells/ $\mu \mathrm{L})$, $n / N(\%)$

$0-100$ 80/199 (40.2)

$100-150$ $34 / 199$ (17.1)

$>150$ 85/199 (42.7)

$\mathrm{IQR}=$ interquartile range.

*Not all information was available for all the subjects.

Table 2. Treatment outcomes after 60 months on treatment $(N=381)$

\begin{tabular}{ll}
\hline Outcome & $\boldsymbol{n}(\%)$ \\
\hline Active & $156(40.9)$ \\
\hline Died & $15(3.9)$ \\
Defaulted & $19(5.0)$ \\
Lost to follow-up & $76(19.9)$ \\
Stopped ART & $6(1.6)$ \\
Transferred out & $109(28.6)$
\end{tabular}

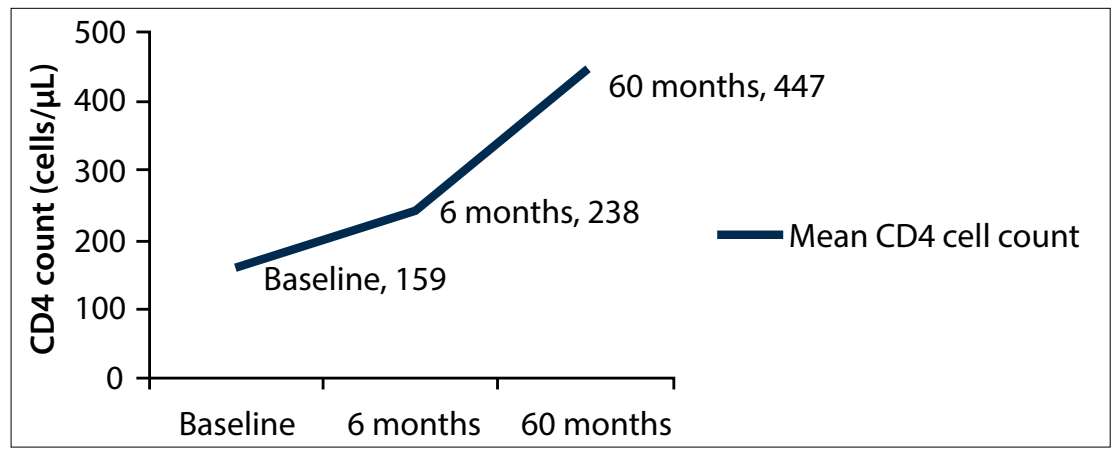

Fig. 1. Change in mean CD4 cell count over 60 months. 


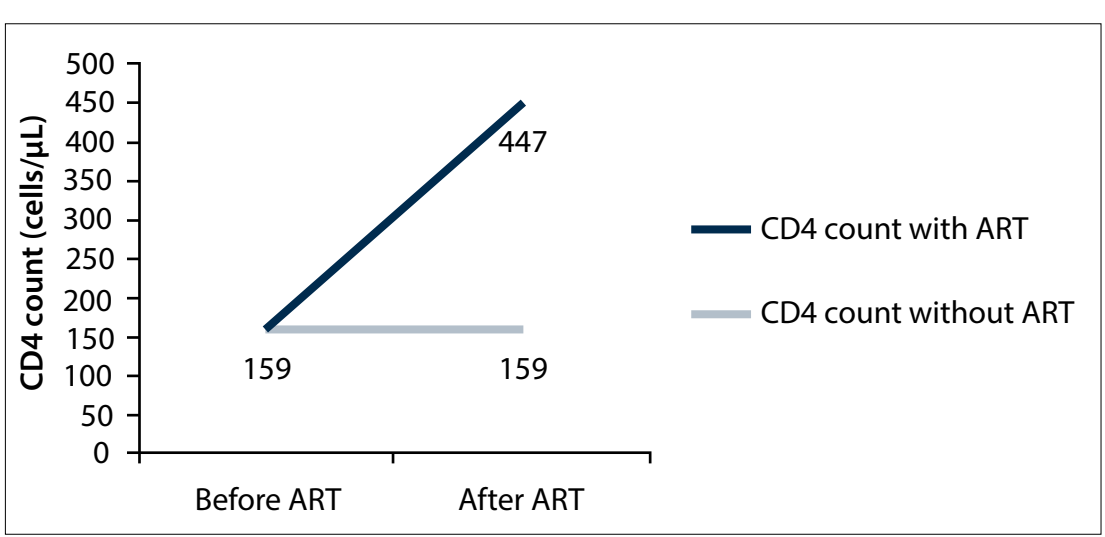

Fig. 2. Impact of ART on immunological response (as indicated by CD4 count), assuming that the CD4 count will not fall further from baseline.

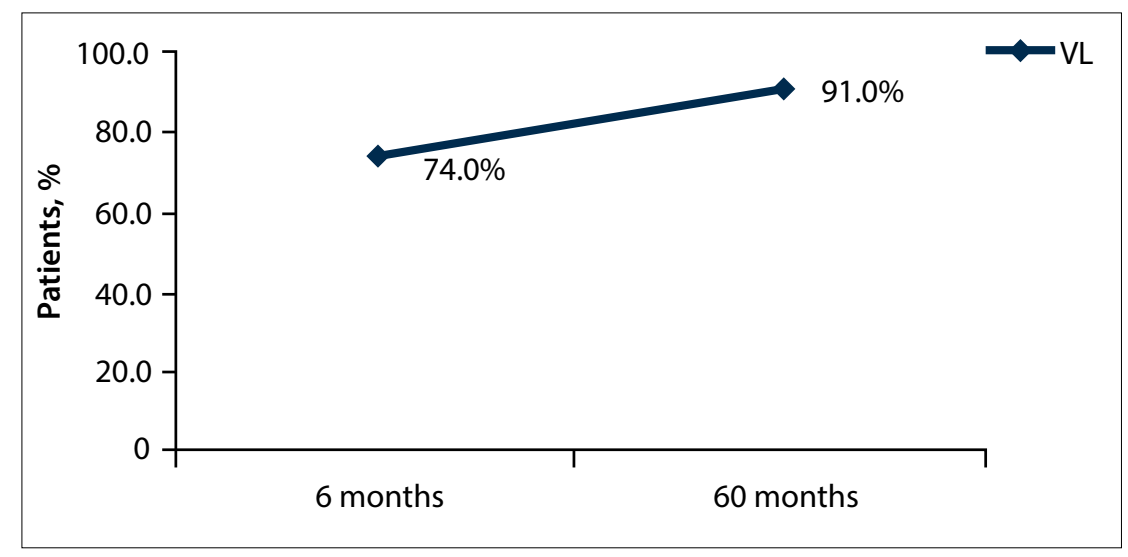

Fig. 3. Proportions of patients with a VL $<400$ copies/mL at 6 and 60 months.

greater CD4 count increase than the other age groups, while patients aged $>42$ years had a lower increase than the other age groups. Patients who had a relatively high baseline CD4 count tended to have a bigger CD4 count change at 6 months than other patients.

Only 29 patients had both baseline and 60-month CD4 counts. Age, gender and baseline CD4 count did not significantly influence the $\mathrm{CD} 4$ count change at 60 months. The mean CD4 count at 60 months was significantly better than that at baseline, with a mean of $447 \mathrm{cells} / \mu \mathrm{L}(p=0.000)$, i.e. a rise of 288 cells $/ \mu \mathrm{L}$ from baseline (measuring records that had both baseline and 60-month results).

Only 79 records had VL results for 6 months and $49 \mathrm{VL}$ results for 60 months. Gender and age did not influence VL outcomes at either 6 or 60 months. VL outcomes at 6 months were poor, with $74.0 \%$ of patients achieving suppression to $<400$ copies/mL, compared with $91.0 \%$ at 60 months (Fig. 3).

\section{Discussion}

Our findings indicate that there needs to be focus on a strategy to improve the quality of SA's ART programme, and thereby ART programme outcomes.
We found that demographic characteristics did not contribute to adverse treatment outcomes. Other studies have previously identified gender and/or age as having an association with adverse outcomes. ${ }^{[1,12]}$ However, our findings are in agreement with those of another study, conducted in Johannesburg, that also found no associations between patient demographics and loss to follow-up. ${ }^{[13]}$ In the current study only CD4 count had a significant association with adverse outcomes combined, which was not the case when loss to follow-up was analysed alone. This means that CD4 count is specifically associated with death on ART, which has also been established in other studies. ${ }^{[1]}$

Our mortality rate of $5.0 \%$ after 60 months on HAART is lower than in previous studies, which have reported $6-11 \%$ mortality rates at $24-36$ months. ${ }^{[1,4,11]}$ Mortality rates may not be accurate, however, as previous findings have revealed that some patients who are recorded as lost to follow-up are in fact dead. While the improvements in CD4 counts at the start of treatment indicate that our mortality rate may not necessarily be inaccurate, tracing of patients who have been lost to follow-up would provide a more accurate rate.
The National Strategic Plan of 2012 - $2016^{[6]}$ stipulates that the retention rate of patients on ART should be at least $80 \%$. Our results show that the programme is currently not retaining more than $60 \%$ of patients after 5 years on treatment. Various factors play a role in poor retention, the major influence in our study being patients who are lost to follow-up. These findings differ from those of a 4-year study in Johannesburg, ${ }^{[14]}$ which showed a retention rate of $74 \%$, while other studies in SA and in other African countries have shown rates of $60-70 \% .^{[1,4,1,1,13]}$

To evaluate the impact of ART on the immune system, we compared CD4 counts before and after exposure to treatment. The changes observed can be attributed to the ART programme alone, as CD4 counts have never been shown to improve on their own without treatment. Since a low CD4 count is associated with mortality, the ART programme therefore has a high impact in reducing HIV-related mortality.

Females had higher baseline CD4 counts than males, and they had a significantly higher $\mathrm{CD} 4$ count increase at 6 months. Other studies have also established that males have lower CD4 counts, both at baseline and during therapy ${ }^{[1,15]}$ There have been many suggestions as to why this should be so, such as males starting treatment later than females, together with biological and sociocultural factors. ${ }^{[2,14]}$

We also found that patients who started treatment with a relatively high $\mathrm{CD} 4$ count had a greater rise in CD4 count at 6 months. The SA ART initiation eligibility criteria have recently changed to a CD4 count of 350 instead of 200 cells/ $\mu \mathrm{L}$, so CD4 count increases are likely to be much higher in the future.

\section{Study limitations}

Our study had a number of limitations. Firstly, we analysed data that had already been collected as routine data. There were lots of missing data, which had a serious impact on the analysis of measurements such as CD4 count and VL. It was therefore difficult to analyse these measurements at intervals of 12 , 24, 36 and 48 months, as had initially been intended. Secondly, we could not do further follow-up on whether the causes of reported deaths were HIV related or not. Thirdly, because of time constraints we could not trace at least a sample of those lost to follow-up, to establish whether they were still alive and obtaining treatment elsewhere, or had died.

\section{Conclusions}

The findings of this study have revealed important health systems-related issues that need to be strengthened in order to improve the outcomes of the national ART 
programme. According to the $\mathrm{WHO}$, the health information system is one of the six health systems building blocks. ${ }^{[15]}$ Poor data quality has a negative impact on the routine monitoring of the programme, on patient management and follow-up, and even on programme evaluation. It is important to have an efficient, up-to-date health information system for this programme in order to improve research output, both for students and for researchers.

Proper records of patient transfers to other facilities are important, and more important are ways to find out whether patients do in fact report to the facilities they are transferred to. Doing so will limit the uncertainty experienced in classifying transferred-out patients as still alive and on treatment.

The study showed that the ART programme has managed to maintain high VL suppression and to improve immunity in patients who are immunocompromised. Both these responses indicate that the programme is successful. ${ }^{[4,5]}$ Further studies that follow each patient reported as lost to follow-up are recommended. Strengthening of patient support and tracing is likely to reduce the number of such patients. Proper data quality management will improve the monitoring of care, and thereby also the programme outputs and outcomes.

Disclaimer. The views expressed in this article are those of the authors and not an official position of the institution to which they are affiliated.

Acknowledgements. We thank the Foundation for Professional Development for allowing NM to use its SOZO database for this project. Ms Suzanne Johnson was particularly helpful in the use of the database. We thank Prof. Samuel Manda of the South African Medical Research Council for statistical assistance, Ms Barbara English of the University of Pretoria for language editing, Tshwane district for granting us permission to conduct the study in its facilities, and the National Institute for Occupational Health for all its support in completing this article.

\section{References}

1. Cornell M, Grimsrud A, Fairlall L, et al. Temporal changes in programme outcomes among adult patients initiating antiretroviral therapy across South Africa, 2002-2007. AIDS 2010;24(14):2263-2270. DOI:10.1097/QAD.0b013e32833d45c5

2. Kolola-Dzimadzi R, Chen SC, Yu JK, et al. Increased mortality of male adults with AIDS related to poor compliance to antiretroviral therapy in Malawi. Trop Med Int Health 2008;13(4):513-529. DOI:10.1111/j.1365-3156.2008.02029.x

3. Karcher H, Omondi A, Odera J, Kunz A, Harms G. Risk factors for treatment denial and loss to follow-up in an antiretroviral treatment cohort in Kenya. Trop Med Int Health 2007;12(5):687-694. DOI:10.1111/j.1365-3156.2007.01830.x

4. Assefa Y, Kiflie A, Tesfaye D, et al. Outcomes of antiretroviral treatment program in Ethiopia: Assefa Y, Kiflie A, Tesfaye D, et al. Outcomes of antiretroviral treatment program in Ethiopia:
Retention of patients in care is a major challenge and varies across health facilities. BMC Health Serv Res 2011;11(1):81. DOI:10.1186/1472-6963-11-81

5. Klausner JD, Serenata C, O'Bra H, et al. Scale-up and continuation of antiretroviral therapy in South African treatment programs, 2005-2009. J Acquir Immune Defic Syndr 2011;57(3):e92. DOI:10.1097/ QAI.0b013e3182067d99

6. National Department of Health. National Strategic Plan 2012-2016. 2011. http://www.doh.gov.za/docs/ Stratdocs/ (accessed 7 February 2012).

7. Nglazi MD, Lawn SD, Kaplan R, et al. Changes in programmatic outcomes during 7 years of scale-up at a community-based antiretroviral treatment service in South Africa. J Acquir Immune Defic Syndr 2011;56(1):e1-8. DOI:10.1097/QAI.0b013e3181ffobdc

8. Lawn SD, Landon Myer L, Harling G, Orrel C, Gail-Bekker L, Wood R. Determinants of mortality and nondeath losses from an antiretroviral treatment service in South Africa: Implications for program evaluation. Clin Infect Dis 2006;43:770-776. DOI:10.1086/507095

9. National Department of Health. Clinical guidelines for the management of HIV and AIDS in adults and adolescents. 2010. http://www.health-e.org.za./news/article (accessed 10 December 2011).

10. Fox MP, Rosen S. Patient retention in antiretroviral therapy programs up to three years on treatment in sub-Saharan Africa, 2007-2009: Systematic review. Trop Med Int Health 2010;15(s1):1-15.
in in sub-Saharan Africa, 2007-2009: S
DOI:10.1111/j.1365-3156.2010.02508.x

11. Boyles TH, Wilkinson LS, Leisegang R, Maartens G. Factors influencing retention in care after starting antiretroviral therapy in a rural South African program. PLoS One 2011;6(5):e19201. DOI:10.1371/ journal.pone.001920

12. Hawkins $\mathrm{C}$, Chalamilla G, Okuma J, et al. Sex differences in antiretroviral treatment outcomes among HIV-infected adults in an urban Tanzanian setting. AIDS 2011;25(9):1189-1197. DOI:10.1097/ QAD.0b013e3283471deb

13. Sanne IM, Westreich D, Macphail AP, Rubel D, Majuba P, Van A. Long term outcomes of antiretroviral therapy in a large HIV/AIDS care clinic in urban South Africa: A prospective cohort study. J Int AIDS Soc 2009;12:38. DOI:10.1186/1758-2652-12-38

14. Dou Z, Xu J, Jiao JH, et al. Gender difference in 2-year mortality and immunological response to ART in an HIV-infected Chinese population. PLoS One 2011:6(8):e22707. DOI:10.1371/journal. pone. 0022707

15. World Health Organization. Monitoring the building blocks of health systems: A handbook of indicators and their measurement strategies. WHO, 2010. http://www.who.int.healthinfo/systems/ monitoring/en (accessed 5 January 2012).

Accepted 16 November 2015. 